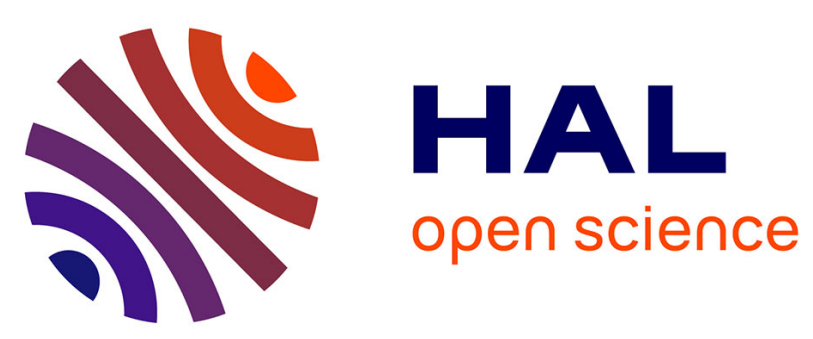

\title{
Domain Structure and Magnetization Reversal Micromechanisms in Quasi-Two-Dimensional Exchange-Biased Nanomagnetics
}

\author{
V. Gornakov, I. Shashkov, M. Lebyodkin, T. Lebedkina
}

\section{- To cite this version:}

V. Gornakov, I. Shashkov, M. Lebyodkin, T. Lebedkina. Domain Structure and Magnetization Reversal Micromechanisms in Quasi-Two-Dimensional Exchange-Biased Nanomagnetics. Physics of the Solid State, 2018, 60 (11), pp.2222-2230. 10.1134/S1063783418110082 . hal-02357167

\section{HAL Id: hal-02357167 \\ https://hal.science/hal-02357167}

Submitted on 10 Jun 2021

HAL is a multi-disciplinary open access archive for the deposit and dissemination of scientific research documents, whether they are published or not. The documents may come from teaching and research institutions in France or abroad, or from public or private research centers.
L'archive ouverte pluridisciplinaire HAL, est destinée au dépôt et à la diffusion de documents scientifiques de niveau recherche, publiés ou non, émanant des établissements d'enseignement et de recherche français ou étrangers, des laboratoires publics ou privés. 
Physics of the Solid State 60(11) (2018) 2222-2230

DOI: $10.1134 / S 1063783418110082$

Pleiades Publishing, Ltd., 2018. https://www.pleiades.online/en/journal/physsost/

Translated from Russian: Fizika Tverdogo Tela 60(11) (2018) 2181-2189

\title{
Domain Structure and Magnetization Reversal Micromechanisms in Quasi-Two-Dimensional Exchange-Biased Nanomagnetics
}

\author{
V. S. Gornakov ${ }^{a, *}$, I. V. Shashkova , M. A. Lebyodkin", and T. A. Lebedkina ${ }^{b}$ \\ "Institute of Salid Slate Physics, Russian Academy of Sciences, Chemogolovka, Mascow oblast, 142432 Russia \\ ${ }^{b}$ Labonatoire d'Etude des Microstructures et de Mecanique des Matériaux, Université de Lomaine, \\ CNRS, Arts et Metiers ParisTech, Metz, Frunce \\ *e-mail: gomakov@issp.ac.nu \\ Received May 14, 2018
}

\begin{abstract}
The domain structure and magnetization reversal mechanisms are studied in quasi-two-dimensional exchange-biased $\mathrm{NiFe} / \mathrm{FeMn}$ and $\mathrm{NiFe} / \mathrm{NiO}$ nanomagnetics, using a complex method of magnetooptical indicator films and acoustic emission. The presence of the axial dispersion of unidirectional anisotropy in grains of the antiferromagn ftic layer is shown to determine the statistic distribution and chirality of spin springs near the interface. The acoustic emission signals caused by the excitation of elastic Lamb waves upon the magnetization reversal of $\mathrm{NiFe} / \mathrm{NiO}$ heterostructure are found. The coercive force of these systems is duc to irreversible processes to overcome potential barriers that are induced by the formation of spin springs with different chirality, localized in the antiferromagnetic near the ferromagnetic-antiferromagnetic boundary.
\end{abstract}

\section{INTRODUCTION}

Thin-film ferromagnetics (FMs) coupled with antiferromagnetic (AFM) by the exchange interaction at their interface are distinguished from the bulk ferromagnetics by the broken magnetic symmetry consisting in the energy nonequivalence of magnetization switching in opposite directions. A FM/AFM heterostructure is characterized by unidirectional (exchange) anisotropy which is manifested by the shifted and broadened hysteresis loop of the FM layer [1]. A key role in the formation of exchange anisotropy is assigned to redistribution and evolution of spins localized in the antiferromagnetic near the interface [2-5]. Nowadays, there are various well-known models interpreting the hysteresis loop shift $[2,3,6-8]$ as well as the increase in coercive force $[8-11]$ in these heterostructures. The magnetization reversal of an exchange-biased FM-layer is shown to depend on the nucleation and evolution of the exchange spin spring (partial domain wall) in the antiferromagnetic close to the interface. The concept of spin spring implies its twisting (untwisting) in the AFM-layer, while the FM-layer undergoes the magnetization reversal from (to) the ground state $[2,4,5,11-14]$. Furthermore, the hysteresis loop shift is due to reversible reorientation processes of spins in the FM-layer and the related spins in the AFM-layer $[2,3,7]$. In turn, the hysteresis loop broadening is on account of the irreversible redistribution of these spins $[8,9,11,14]$. In particular, the latter arise owing to the fact that, in real bilayer struc- tures, both intralayer and interface imperfections can induce the formation of exchange springs with oppositely directed twisting, i.e., different chirality, likewise two-dimensional domain walls in the bulk ferromagnetics $[15,16]$, or spin springs in exchange-coupled magnetohard/magnetosoft ferromagnetic layers [1720]. In this case, multidirectional twisting of spins in the neighboring areas of the heterostructure together with a field applied against the saturation magnetization favors the formation of local potential barriers in the form of 360-degree domain boundaries or metastable areas with incomplete inversion of the magnetic moments. Overcoming these barriers requires additional energy; i.e., the magnetic field must be amplified, resulting in increased coercive force. On the other hand, thermal fluctuations of spins from the metastable state to the energetically sustainable one determine the observed magnetic aftereffect [21-23] To describe regularities of the coercive force formation and the aftereffect in real FM/AFM magnetic heterostructures, a series of models considering the irreversible switching of the order parameter in polycrystal grains in the AFM-layer upon the magnetization reversal of a ferromagnetic was proposed in $[8,9,11]$. Quasi-two-dimensional exchange-biased FM/AFM nanomagnetics with a polycrystalline AFM layer are the structures, in which the axial dispersion of anisotropy in grains exerts the strongest influence on the irreversible magnetization reversal processes. The deviation of the distribution of AFM spins from the equilibrium configuration leads to the metastable state 
that manifests itself by the so-called training effect. It consists in the dependence of hysteresis loop parameters upon the sequential cyclic variation of the magnetic field $[21-23]$ and can be attributed to the longterm spin relaxation in the AFM-layer. Since the domain structure transformation in FM/AFM films makes the mechanisms of coercivity formation $[8,9$, 11] and the training effects in them clearer, investigating the behavior of magnetic moments in the FM-layer and magnetoelastic properties in the AFMlayer is of great importance [21-23]. The present work aims to be a study of the domain structure and the magnetization reversal micromechanisms in quasitwo-dimensional exchange-biased nanomagnetics via the magnetooptical visualization of magnetization distribution in a FM-layer and the recording of ultrasonic excitations (acoustic emission) in an AFM-layer under the DC and $\mathrm{AC}$ magnetic fields.

\section{EXPERIMENTAL}

Samples were the polycrystalline $\operatorname{NiFe}(16 \mathrm{~nm}) /$ $\mathrm{FeMn}(30 \mathrm{~nm})$ and $\mathrm{NiFe}(10 \mathrm{~nm}) / \mathrm{NiO}(50 \mathrm{~nm})$ films grown via the magnetron and ion-beam sputtering, respectively, onto the silicon substrates oxidized in air. The unidirectional anisotropy was applied under the external magnetic field with $H=300$ Oe upon the growth of samples. The antiferromagnetic in the second heterostructure was $\mathrm{NiO}$ nickel oxide with significant magnetostriction that favors the formation and recording of magnetoelastic excitations in the heterostructure while subjected to magnetization reversal. The ferromagnetic was $\mathrm{Ni}_{79} \mathrm{Fe}_{21}$ permalloy with a magnetostriction tending to zero. Thus, the FM film gives no magnetoelastic contribution to a signal acquired from samples; i.e., any manifestation of this signal will be exclusively due to the transformation of a system of spins localized in the AFM-Iayer.

The microscopic study of magnetization reversal in the FM/AFM heterostructure was implemented through the visualization of stray magnetic fields in a reflected linearly polarized light using a magnetooptical indicator film technique (MOIF) $[19,24]$. The indicator was a thin film of bismuth-doped ironyttrium gamet with an in-plane magnetization, which exhibits the large Faraday effect. When the indicator is placed onto a surface of the FM/AFM-sample, the normal components $H_{1}$ of local stray fields of the FMlayer induce the appropriate local deviation of magnetization of the indicator from its plane. Owing to the double Faraday effect, the normal component of the magnetic moment of the indicator initiates the rotation of the polarization plane of the light reflecting from the surface adjacent to the sample and, depending on the value and polarity of the local field $H_{1}$, is visualized in the form of dark or light areas against the grey background of the magnetooptical (MO) portrait of the sample. In order to elucidate regularities of the formation of exchange springs, the MO pattern was recorded in the rotating magnetic field. To analyze the magnetization in the plane of a sample, the latter was pierced to obtain a through hole with a diameter of $300 \mu \mathrm{m}$ (Fig. Ia). The direction of the effective magnetization $\vec{M}$, forming under external magnetic fields, was determined from the orientation of a symmetry axis (shown with arrow) of the magnetooptical pattern formed by the stray field components at the edge of the hole. The quantitative characteristic of $M$ was the averaged intensity of the magnetooptical signal $I_{A}=$ $\left(I_{L}+I_{R}\right) / 2$, where $I_{L}$ and $I_{R}$ are the maximum intensities at the "dark" and "light" edges. This value is defined by the angle of the Faraday rotation of the polarization of the light, proportional to the perpendicular field $H_{\perp}$ at the edge of the hole and, therefore, to the magnetization averaged over sample thickness. The hysteresis loops were measured using a vibrating sample magnetometer in the room-temperature field oriented along the axes of FM/AFM heterostructures.

The elastic excitations induced by the deformation of samples in polycrystalline heterostructures with (a)

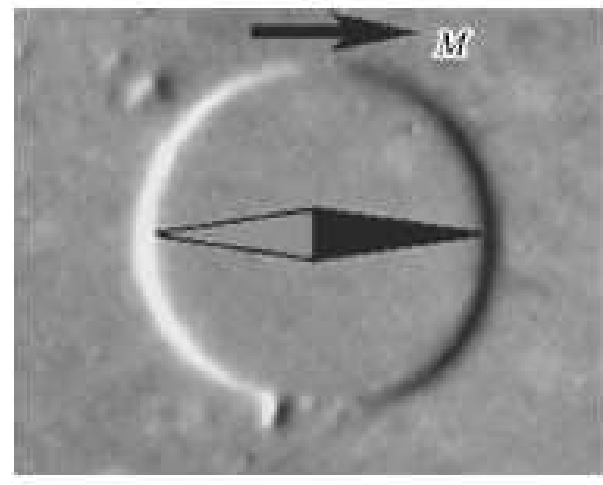

(b)

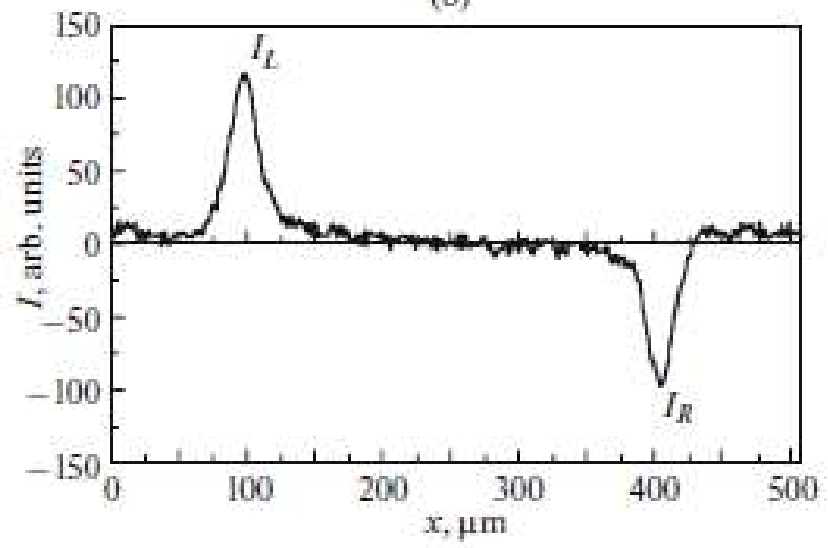

Fig. 1. (a) MO portnit of NiFe/FeMn sample and (b) MO signal intensity along the arrow of a compass. 

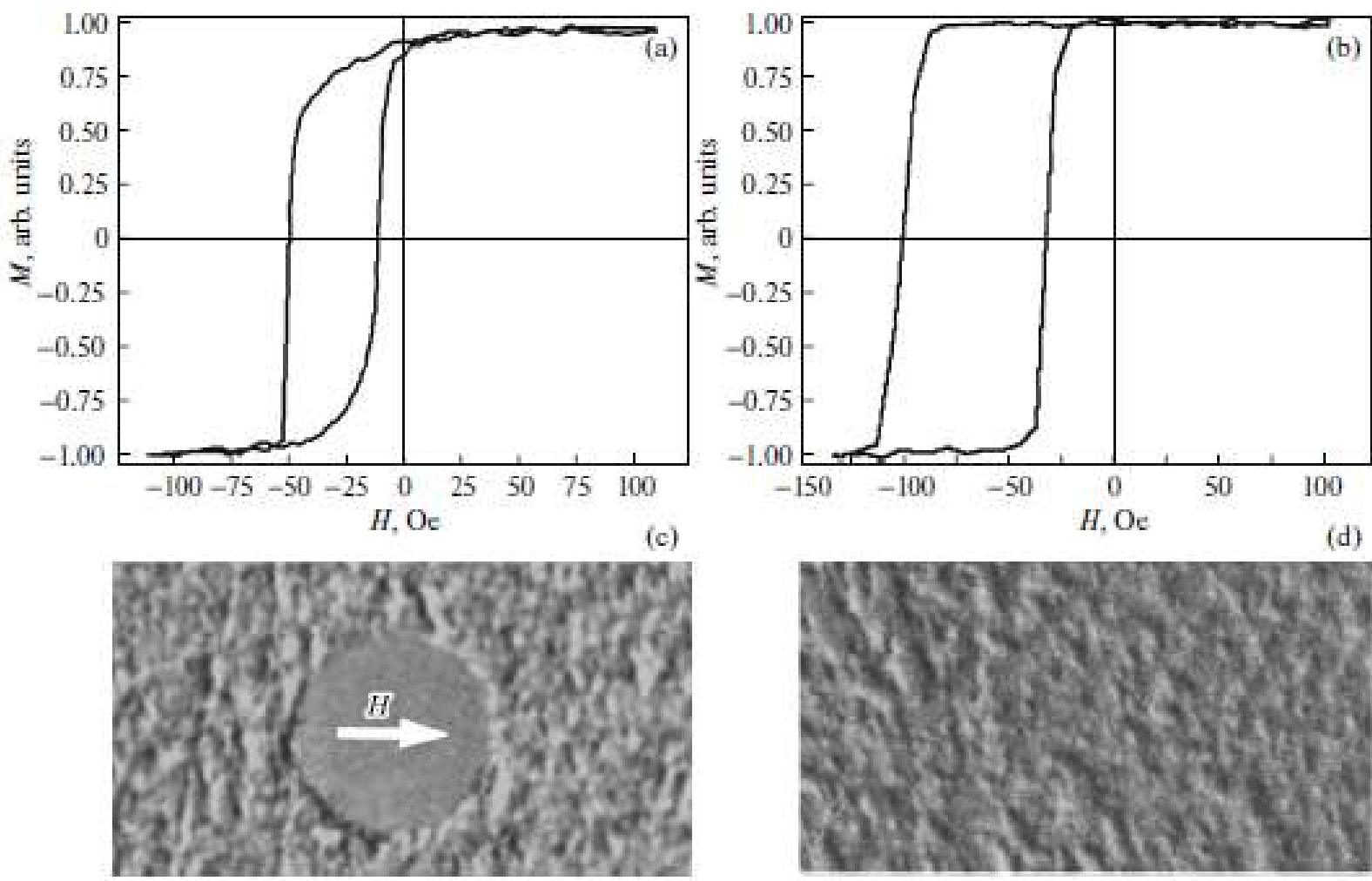

Fig. 2. (a) and (b) Hysteresis loops and (c) and (d) MOIF images of the domain structure of (a, c) NiFe/ FeMn and (b, d) $\mathrm{NiFe} / \mathrm{NiO}$ heterostructures exposed to magnetiz ation reversal along the unidirectionan anisotropy axis. The magnetic field in the MO recording was (c) 51.0 and (d) $103.0 \mathrm{Oe}$.

magnetorestriction antiferromagnetic NiO layers were recorded using the acoustic emission (AE) technique. Being suitable for measuring the plasticity $[25-27]$, this technique was adapted to highlight the magnetoelastic effects in films [28]. A sample and a piezoelectric sensor placed on the sample surface through a lubricant buffer for the better contact with a surface were put in a uniform magnetic field. The working frequency band of the sensor was in a range of $200-900$ $\mathrm{kHz}$. The captured AE signal was preamplified by 60 $\mathrm{dB}$ and recorded in the memory of the computer with a sampling frequency of $2 \mathrm{MHz}$. To verify the acoustic contact between the sample and the sensor and to get the pulse response characteristics of a sensor, there was performed a conventional test (Hsu-Nielsen source) [29], consisting in the acute single mechanical effect on a table near the sample with a sensor. The pulse increases with a time of about several microseconds and is damped within a few milliseconds. Its frequencies are below $50 \mathrm{kHz}$.

\section{RESULTS AND DISCUSSION}

Figure 2 displays the hysteresis loops of heterostructures and the characteristic domain structure upon their magnetization reversal along the axis of unidirectional anisotropy. The bias fields of hysteresis loop and coercivity of the $\mathrm{NiFe} / \mathrm{FeMn}$ and $\mathrm{NiFe} / \mathrm{NiO}$ heterostructures were 33 and $19 \mathrm{Oe}$ and 67 and $31 \mathrm{Oe}$, respectively.

The magnetization switching in $\mathrm{NiFe} / \mathrm{FeMn}$ (Fig. 2c) and $\mathrm{NiFe} / \mathrm{NiO}$ (Fig. 2d) occurs as a result of inhomogeneous magnetization reversal with formed irregular domain structure. So, the domain structure visualization reveals that magnetization reversal in these structures is mainly due to microscopically nonuniform spin states.

In order to elucidate the contribution of the exchange spring to the hysteresis properties in polycrystalline FM/AFM bilayer films, the domain structure was displayed in a $\mathrm{NiFe} / \mathrm{FeMn}$ heterostructure in the magnetic field $H$ rotating in the sample plane (Fig. 3) close to the hole. The rotation angles $\varphi$ of the magnetization $M$ averaged over the FM-layer thickness relative to the axis of unidirectional anisotropy as the functions of rotation angles $\alpha$ of the magnetic field $H$ at its different values are plotted in Fig. 4 .

A dashed line denotes the state at which the magnetization would be cooriented with the field direction. Under a great field $(-60 \mathrm{Oe})$, the magnetization vector $M$ rotates almost synchronously with the field, being slightly behind $H$ at $\alpha<180^{\circ}$ and ahead of $H$ at 
$\alpha>180^{\circ}$ The clockwise and counterclockwise field rotation causes the weak hysteresis. Nevertheless, decreasing the field makes the hysteresis and misalignment between $M$ and $H$ orientations more pronounced. Such a behavior of magnetization is a result of the transformation of FM/AFM domain structure. Figures $3 \mathrm{a}$, 3e displays the MOIF patterns of the magnetization distribution for a counterclockwise rotating field with $H=30.0$ Oe at orientations denoted in Fig. 4 with the same letters. At the onset, the rotation of $M$ from the initial state (Fig. 3a) is almost uniform (Fig. 3b), as can be concluded from a lack of magnetic irregularities in the sample plane and the black-andwhite contrast rotating at the edge of the hole.

When the angle $\alpha$ tends to $180^{\circ}$, a nonuniform MO-contrast arises in the sample (Figs. $3 \mathrm{c}$ and $3 \mathrm{~d}$ ), meaning the nonuniformly redistributed magnetization in the FM-layer. The domain structure transformation is characterized by the changing position of magnetization $M$ relatively $H$. At the onset of the process, the magnetization $\boldsymbol{M}$ was behind the field (Fig. 3c) and began outstripping it with increasing $\alpha$ angle (Fig. 3d). A further increase in $\alpha$ leads to the uniform rotation of magnetization, being coherent to $H$ (Fig. 3e) and its final orientation along the field (Fig. 3a). The magnetization reversal of a sample in the clockwise rotating field is similar, but with a slight hysteresis.

A decrease in the field below a critical value of $H_{\mathrm{CR}}=28$ Oe alters drastically the $\varphi(\alpha)$ dependence. As is seen in Fig. 4, at $H=21.0$ Oe the onset magnetization follows the field with some lag. Nevertheless, unlike the great fields, the angle $\varphi$ declines with increased $\alpha$, and the MO- contrast in the vicinity of $\alpha=180^{\circ}$ becomes also inhomogeneous, evidencing the nonuniform magnetization distribution. Similarly to the large fields, this transformation of magnetization causes the hysteresis in the field rotating in opposite directions. In the weak fields (e.g., at $H=6.0$ Oe in Fig. 4), the magnetic moment slightly deviates from the initial state and induces no pronounced change in MO contrast within a sample.

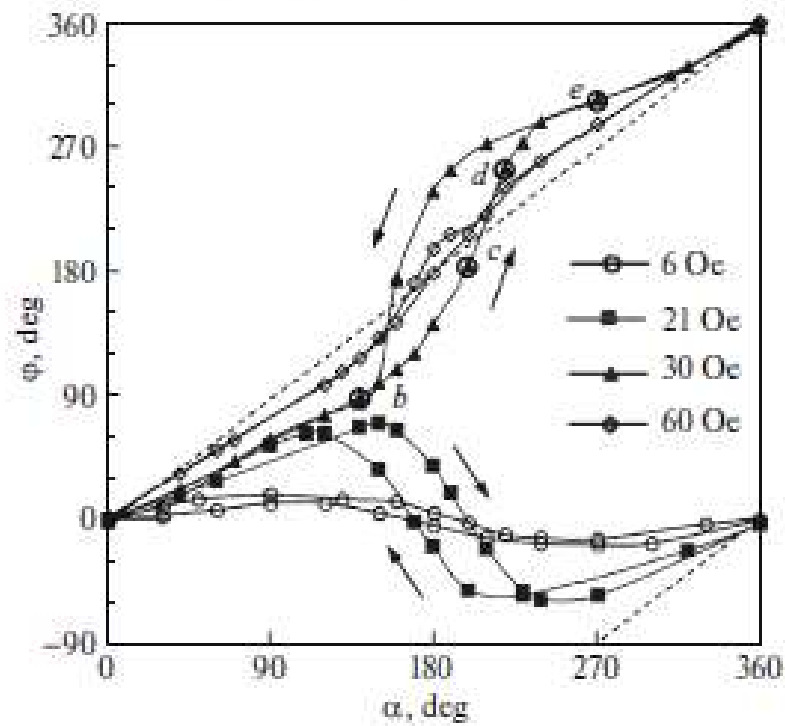

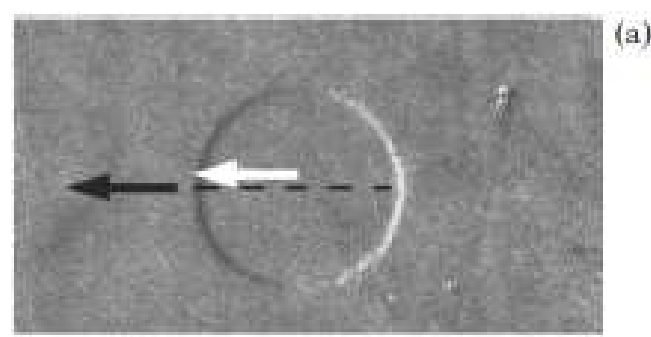

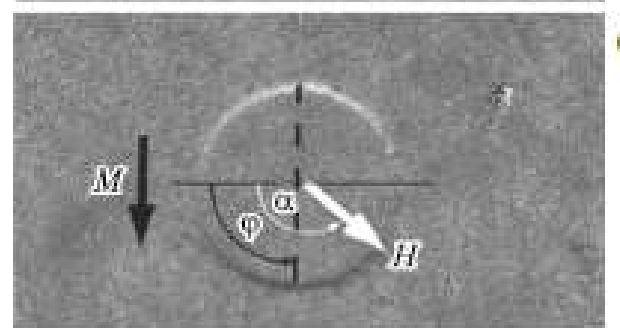

(b)

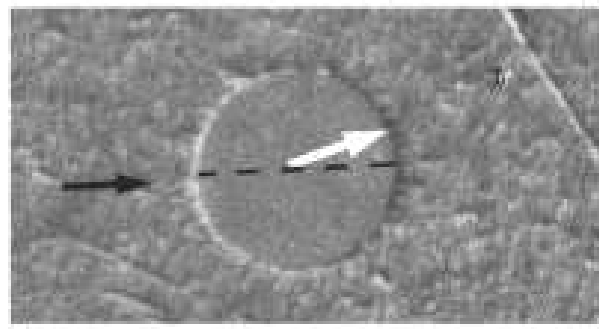

(c)

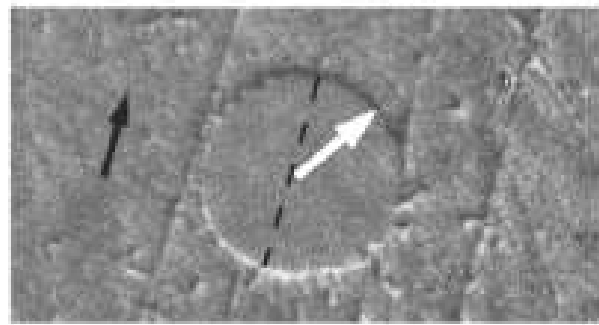

(d)

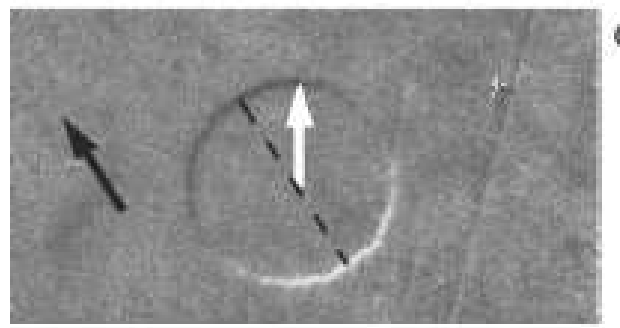

(c)

Fig. 3. MOIF images of the domain structure in the rotating field with $H=30 \mathrm{Oe}$. The moments of recording are denoted with the same letters as in Fig. 4. The white arrow shows the direction of $\mathrm{H}$, and the black amow and the dashed line designate the $\mathbf{M}$ direction found from the MO contrast analysis along the edge of the hole.

Fig. 4. Rotation angle $\varphi$ of magnetization $M$ versus $H$ field $(\alpha$ angle) direction. 

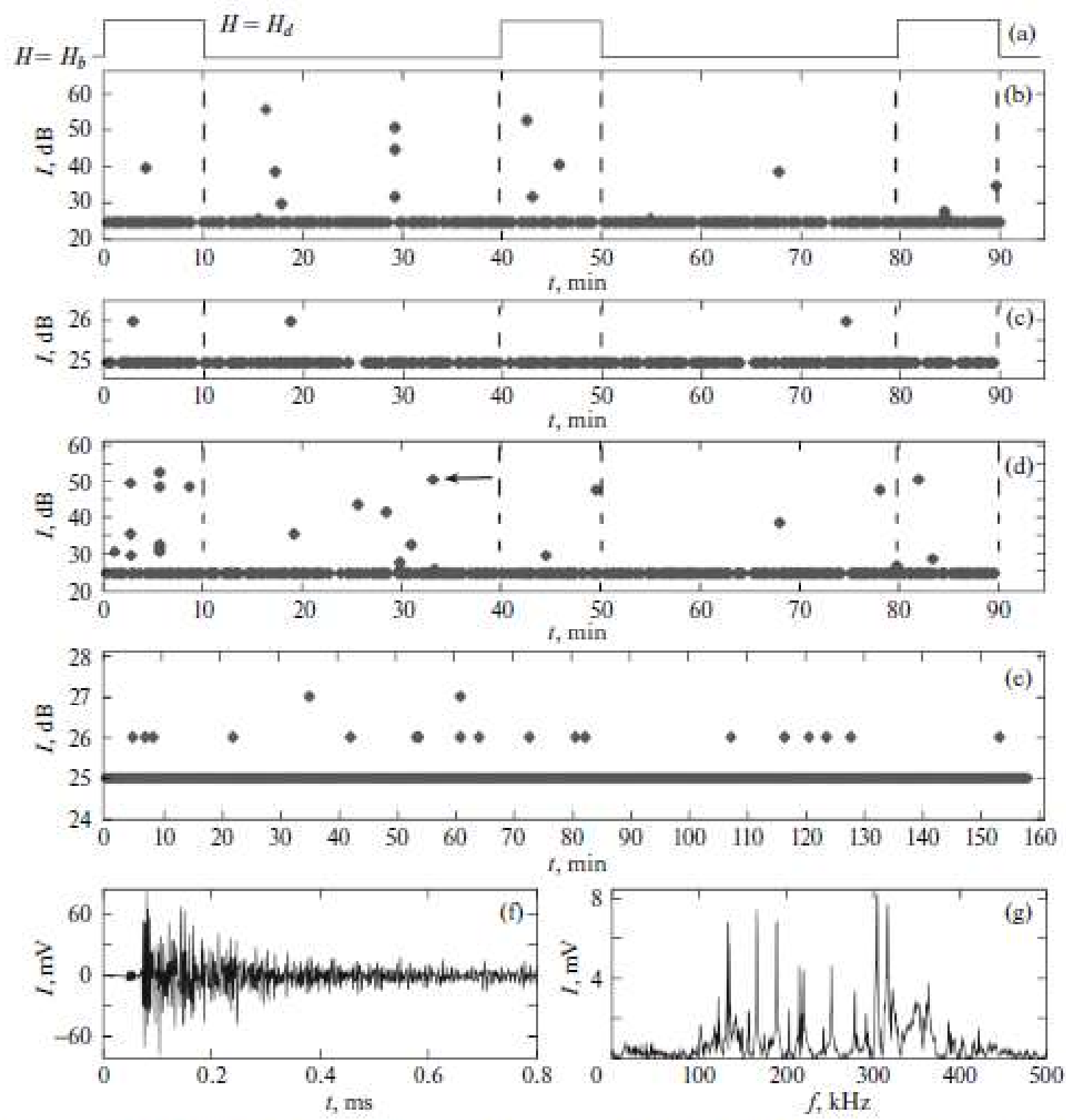

Fig. 5. Acoustic response of $\mathrm{NiFe} / \mathrm{NiO}$ sample $(1 \times 1) \mathrm{cm}$ in (a) pulse magnetic field oriented (b) antiparallel, (c) parallel and (d) perpendicular to the field of unidirectional anisotropy, and in a lack of sample (e). (f) Elastic vibrations and (g) their spectrum correspond to the acoustic signal shown with the arrow on the panel (d).

It is evident that the formation of hysteresis properties of exchange-biased polycrystallinte nanomagnetics is mainly a result of local transformation of the magnetic moments in the FM-layer as well as of switching spin states in the AFM-layer. The experimental study of the real transformation of a system of spins localized in AFM grains via the conventional magnetometry methods is impossible, because the lack of effective magnetization in these grains. The recording of spin state switching in the AFM- layer, induced by the magnetization reversal of the FM/AFM heterostructure is available, if the AFMlayer is a magnetostrictive material, such as nickel oxide that is promising for this purpose. The rapidly changing directions of the magnetic moments in $\mathrm{NiO}$ grains will lead to their abrupt deformations, and, consequently, to the excitation of shock elastic waves whose acoustic emission can be recorded by a piezosensor. To avoid the overlap of the acoustic excitations in the AFM-layer with those in the FM-layer, this must be a material with zero magnetostriction, e.g., $\mathrm{Ni}_{79} \mathrm{Fe}_{21}$ permalloy.

The $\mathrm{Ni}_{7 g} \mathrm{Fe}_{2 !} / \mathrm{NiO}$ samples with areas of $(1 \times 1) \mathrm{cm}$ and $(1 \times 0.5) \mathrm{cm}$ were exposed respectively to rectangular (Fig. 5a) and sawtooth (Fig. 6a) pulse fields. The 
amplitude of pulses $H_{d}=200$ Oe was chosen so that the FM-layer was magnetized to saturation similar to the ground state (at $H_{b}=0 \mathrm{Oe}$ ), which was monitored via the MO measurements. No $\mathrm{AE}$ signals were observed upon the magnetic field switching in a wide range of pulse amplification times from $1 \mathrm{~ms}$ (Fig. 5a) to Is (Fig. 6a). In turn, single acoustic excitations were found in saturated heterostructures under both fields $\left(H_{b}\right.$ and $\left.H_{d}\right)$, when the AE signals were recorded within a sufficiently long wait time after the field switching, for the magnetization switching of samples parallel (Figs. 5b and 6c) and perpendicular (Fig. 5d and $6 \mathrm{~b}$ ) to the unidirectional anisotropy axis.

The series of points in Fig. 5 are attributed to $\mathrm{AE}$ signals recorded in the fields directed antiparallel (Fig. 5b) and parallel (Fig. 5c) to the exchange bias field. In the first case, the acoustic signals were superior to the natural noise of the recording device, measured in a lack of the sample for a long time (Fig. Se). In the second case (Fig. 5c), the probability of AE signal emergence was extremely low.
Once the field was switched on or off, the amplitudes of acoustic excitations and their appearance moments were randomly distributed. In the prolonged cycling of the magnetic field, the probability of $\mathrm{AE}$ event appearance was lower (Fig. Sb), meaning that a system of spins in the AFM-layer tends to a more stable state. As soon as the field pulses cease, the probability of AE excitation is reduced to almost zero with time. It is worth mentioning that the probability of emergence of acoustic excitations depends on the magnetic prehistory of samples. In particular, switching the magnetic field from the parallel orientation (Figs. 5b and 6c) to perpendicular (Figs. 5d and 6b) (or vice versa) increases the probability of appearance of acoustic excitations.

Since the most measured acoustic signals exceed to a large extent the noise of the piezosensor, it can be concluded that the previously described AE events are generated by elastic waves excited in the $\mathrm{NiFe} / \mathrm{NiO}$ heterostructure. Figures $5 \mathrm{f}$ and $6 \mathrm{~d}$ display two typical damping oscillations of $\mathrm{AE}$ signals, recorded at the
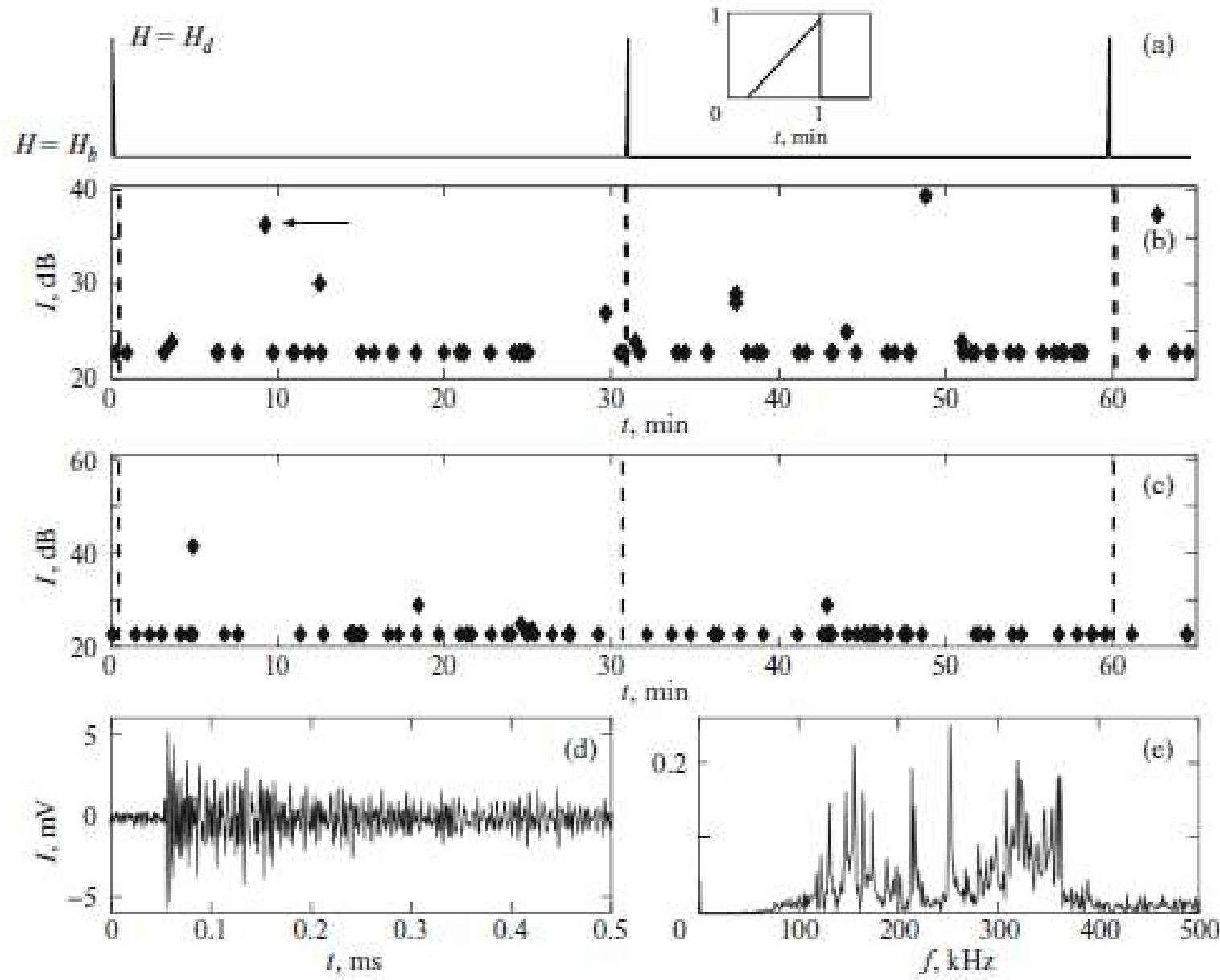

Fig. 6. Acoustic response of $\mathrm{NiFe} / \mathrm{NiO}$ sample $(1 \times 0.5) \mathrm{cm}$ in (a) sawtooth magnetic field oriented (b) pemendicular and (c) parallel to the field of unidirectional anisotropy. (d) Flastic vibrations and (e) their spectrum correspond to the acoustic signal shown with the arrow on the panel (d). 
moments (shown with arrows in Figs. 5d and 6b) after removal of a perpendicular field for two samples with different sizes and shapes. Figures $5 \mathrm{~g}$ and $6 \mathrm{e}$ display the corresponding Fourier spectra. In both cases, the elastic vibration spectra exhibit the identical set of harmonics in a range of 100 to $400 \mathrm{kHz}$ at any shape and size of samples, and the attenuation times are within tenths of milliseconds, which is beyond the natural vibration frequencies $(0-50 \mathrm{kHz})$ and attenuation times (several milliseconds) of a sensor response to the standard test (Hsu-Nielsen source). It should be noted that the amplitudes of the recorded spectra are strongly different, despite a quite similar structure of harmonics. The analysis of a large amount of measured $\mathrm{AE}$ signals reveals that the $\mathrm{AE}$ events with changing amplitudes, acquired under sundry fields for samples with different sizes and at various moments of time and at different areas give the identical frequency spectra. It can therefore be pointed out that the Fourier spectra in Figs. $5 \mathrm{~g}$ and $6 \mathrm{e}$ reflect the properties of the ultrasonic wave excited in a studied medium as a result of its impact deformation.

The acoustic sensor responds to elastic surface waves with vertical polarization normal to a surface. Such a polarization in thin slabs and films may lead to the excitation of the so-called Lamb waves [30-32]. Their parameters are determined by the film thickness and the material structure, i.e., by the anisotropy of elastic interactions [33]. Thus, the presence of elastic vibration modes in the Fourier spectra testifies to the excitation of the Lamb waves in the heterostructure. Taking into account the almost zero magnetostriction in $\mathrm{NiFe}$, it can be inferred that the excitation of elastic vibration occurs in the antiferromagnetic $\mathrm{NiO}$ layer as a result of spin switching initiated by the rotation of spins in the ferromagnetic NiFe layer due to the exchange interaction of FM and AFM spins at the interface.

The nucleation of domains and the motion of domain walls in a two-phase film (Fig. 2) cause nonuniform spin rotation in both FM and AFM-layers near the interface $[2,14,15]$. The coherent rotation of FM spins in the magnetic field is accompanied by the formation of local spirals with right- and/or lefthanded spin twisting in AFM grains because the dispersion of their axial anisotropy (Fig. 7a). This brings forth the metastable states that are characterized by the formation of $360^{\circ}$ topological domain boundaries (Fig. 7b). The potential barriers arising to make the heterostructure be relaxed to a more stable state cannot be overcome if no additional energy is applied. The presence of barriers favors the irreversible processes of abrupt transition between two degenerate magnetization states of AFM grains $[9,11]$ and the excitation of elastic waves, because of the magnetostriction. It should be noted that these transitions must be thermally activated and depend on the prehistory and the amount of field pulse repetitions.
The present model admits two specific mechanisms [9]. The first of them assumes that the effective average angle of some exchange spin springs in antiferromagnetic exceeds the critical value [11] and the AFM grains pass to another state via the overcoming of barriers. Most processes are due to the movement of hybrid FM/AFM domain walls [5], and their statistical averaging gives no contribution to the magnetostrictive response, while measuring the $\mathrm{AE}$ signals upon the magnetization reversal of $\mathrm{NiFe} / \mathrm{NiO}$ heterostructures. The applied energy expenditure and the appropriate hysteresis effect are the result of ineversible spin switching in AFM grains. Nevertheless, some high energy barriers cannot be overcome within or immediately after switching. This ensures that a sample remains in the metastable state with the energy determined by grains with twisted spins. The return to a stable state requires the thermally activated untwisting processes that are implementable through the nucleation and motion of domain $T$-walls, which will lead to spin rotation in $\mathrm{NiO}$ grains and, consequently, reorientation of the anisotropy axis and magnetostrictive deformation of grains. The abrupt change in size of isolated grains, caused by their deformation, will result in the emergence of a shock wave that may induce the above observed Lamb waves.

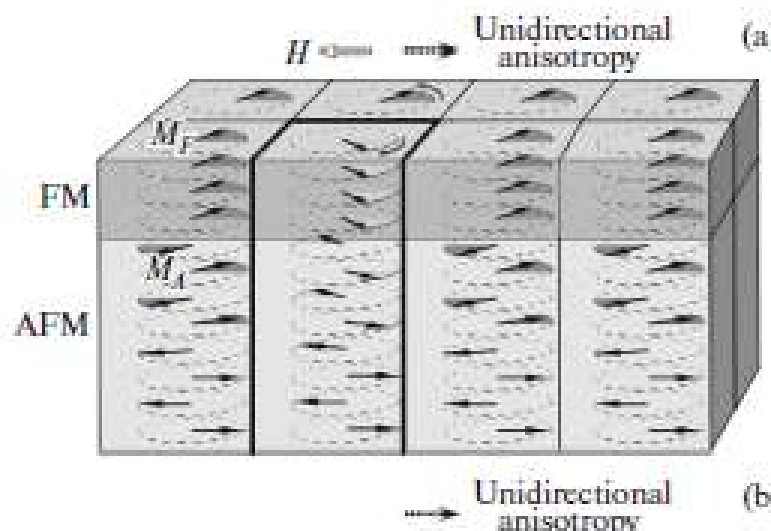

(a)

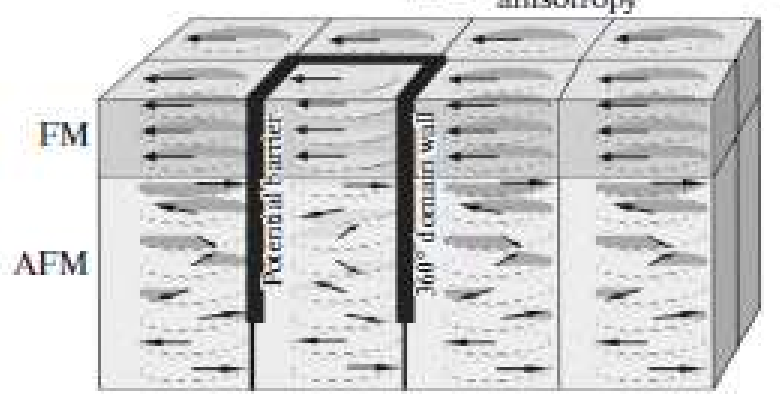

Fiz. 7. Schematic of magnetic moments distributed in FM- and AFM-layers of the exchange-biased heterostructure containing the grain with inverse spin spinal (a) before and $(b)$ after magnetization reversal. 
The second feasible mechanism of elastic wave excitation is a process of paired recombination of $T$-walls in twisted $\mathrm{NiO}$ grains [32]. The $T$-walls are prone to easy nucleation, displacement and annihilation as a result of the emergence of internal stresses upon the deformation of some grains with spin rotation in them. The elastic energy accumulated in two walls can be released during their annihilation in the form of a pulse of an elastic shear wave that will spread as a Lamb wave along the NiO layer. The magnetoelastic excitations arising in magnetization reversal of $\mathrm{NiFe} / \mathrm{NiO}$ heterostructures convincingly show that coercivity of these structures is associated with irreversible overcoming of potential barriers generated by the different-chiral spin springs localized in antiferromagnetic in the vicinity of the FM-AFM interface.

The axial dispersion of unidirectional anisotropy in grains determines the statistical distribution and chirality of spin springs near the interface under the magnetic fields. For these heterostructures, subjected to switching along the axis of unidirectional anisotropy, the opposite spin twisting is equiprobable and the effective magnetization keeps its orientation unchanged (Fig. 2b). The chiral symmetry breaks in the rotating magnetic fields. The anisotropy of grains exposed to large fields is overcome and there is a quasi-continuous rotation of magnetization (Fig. 4 , see the curve at $H=60 \mathrm{Oe}$ ); i.e., there is the formation of monochiral exchange springs. Applying the weak fields to grains brings them to a slight perturbation that is incapable to overcome anisotropy in most grains (Fig. 4 , see the curve at $H=6 \mathrm{Oe}$ ). In the case of intermediate fields rotating in one part of grains, the anisotropy is overcome and the spin spring twisting direction matches that of the field rotation. Another part of grains remains with insuperable anisotropy, and spin springs in them are twisted in the opposite direction, returning to the initial state at the end of the field rotation cycle or being in the metastable state. The volume ratio of grains which may follow the field to that of grains which are resistant to the field must depend on the field strength. The change in $\varphi(\alpha)$ dependence with a field deviating from a value of $H=H_{C R}$ confirms it (Fig. 4, curves at $H=30$ and $21 \mathrm{Oe})$.

\section{CONCLUSIONS}

The domain structure visualization in a $\mathrm{NiFe} / \mathrm{FeMn}$ heterostructure together with a change in the $\mathrm{AE}$ signal in a $\mathrm{NiFe} / \mathrm{NiO}$ heterostructure revealed that magnetization switching in these structures was mainly due to microscopically nonuniform spin states. The recording of acoustic emission in a $\mathrm{NiFe} / \mathrm{NiO}$ heterostructure highlighted the stochastic acoustic signals caused by elastic Lamb waves. These are generated by deformation upon the abrupt chirality switching of magnetic moments in some grains of antiferromagnetic $\mathrm{NiO}$ which exhibits strong magnetostriction.
The appropriate deformation waves are attributed to irreversible changes in the initial spin orientation, which can arise in some antiferromagnetic grains with disordered anisotropy axes during the magnetization reversal of exchange-coupled ferromagnetic/antiferromagnetic structures. Applying the field antiparalle to the unidirectional anisotropy axis was found to favor the formation of spin springs with opposite chirality at different sites of the antiferromagnetic film. Their balance reduces the effective magnetization of the ferromagnetic layer without its rotation. As shown, the deviation of the field from the anisotropy axis makes the balance and, consequently, the magnetization rotation violated. If the rotating in-plane magnetic field is $H<H_{\mathrm{CR}}$, then the magnetization rotation is inverse, otherwise it is quasi-unidirectional and corresponds to a monochiral spin spring in the AFM layer.

The key factor in increasing the coercivity in this two-phase system is axial dispersion of crystallographic anisotropy in grains of the antiferromagnetic layer near the interface, which leads to the emergence of metastable states with topological barriers separating the areas of the crystal with different-chirality exchange springs.

\section{ACKNOWLEDGMENTS}

This work was supported by the Institute of Solid State Physics, Russian Academy of Science, within the framework of the Program of the RAS "Nanostructures."

\section{REFERENCES}

1. W. H. Meiklejohn and C. P. Bean, Phys, Rev. 102, 1413 (1956); Phys. Rev. 105, 904 (1957).

2. D. Mauri, H. C. Siegmann, P. S. Bagus, and E. Kay, J. Appl. Phys. 62, 3047 (1987).

3. A. P. Malozemoff, Phys, Rev. B 35, 3679 (1987).

4. A. Scholl, M. Liberati, E. Arenholz, H. Ohldag, and J. Stöhr, Phys. Rev. Lett. 92, 247201 (2004).

5. C. L. Chien, V. S. Gornakow, V. I. Nikitenko, A. J. Shapiro, and R. D. Shull, Phys. Rev. B 68, 014418 (2003).

6. J. Nogues, J. Sort, V. Langlais, V. Skumryev, S. Suriñach, J. S. Muñoz, and M. D. Baró, Phys. Rep. 422 , $65(2005)$.

7. F. Radu and H. Zabel, Springer Tracts Mod. Phys. 227, $97(2008)$

8. M. D. Stiles and R. D. McMichael, Phys. Rev. B 59, 3722 (1999).

9. E. Fulcomer and S. H. Charap, J. AppL. Phys, 43, 4190 (1972).

10. T. S. Schulthess and W. H. Butler, Phys. Rev. Lett. 81, 4516 (1998).

11. M. D. Stiles and R. D. McMichael, Phys. Rev. B 63 , 064405 (2001).

12. V. I. Nikitenko, V. S. Gornakov, L. M. Dedukh, Yu. P. Kabanov, A. F. Khapikov, A. J. Shapiro, 
R. D. Shull, A. Chaiken, and R. P. Michel, Phys. Rev. B 57, R8111 (1998).

13. V. I. Nikitenko, V. S. Gornakov, A. J. Shapiro, R. D. Shull, K. Liu, S. M. Zhou, and C. L. Chien, Phys. Rev. Lett. 84, 765 (2000).

14. V. S. Gomakov, Yu. P. Kabanov, O. A. Tikhomirov, V. L. Nikitenko, S. V. Urazhdin, F. Y. Yang, C. L. Chi$\mathrm{cn}$, A. J. Shapiro, and R. D. Shull, Phys. Rev. B 73 , $184428(2006)$.

15. A. P. Malozemoff and J. C. Slonczewski, Maynetic Domain Walls in Bubble Materials (Academic, New York, 1979).

16. A. Hubert and R. Shafer, Magnetic Domains (Springer, Berlin, 1998).

17. R. D. Shull, A. J. Shapiro, V. S. Gomakov, V. I. Nikitenko, J. S. Jiang, H. Kaper, G. Leaf, and S. D. Bader, IEEE Trans. Magn. 37, 2576 (2001).

18. J. S. Jiang, S. D. Bader, H. Kaper, G. K. Leaf, R. D. Shull, A. J. Shapiro, V. S. Gornakov, V. L. Nikitenko, C. L. Platt, A. E. Berkowitz, S. David, and E. E. Fullerton, J. Phys. D 35, 2339 (2002).

19. V. S. Gornakow, Yu. P. Kabanov, V. I. Nikitenko, O. A. Tikhomirov, A. J. Shapiro, and R. D. Shull, J. Exp. Theor. Phys. 99, 602 (2004).

20. S. Mangin, C. Bellouard, S. Andrieu, F. Montaigne, P. Ohresser, N. B. Brookes, and B. Barbara, Phys, Rev. B 70, 014401 (2004).

21. X. Portier, A. K. Petford-Long, A. de Morais, N. W. Owen, H. Laidler, and K. O'Grady, J. Appl. Phys, 87, 6412 (2000).
22. A. N. Dobrynin, F. Maccherozzi, S. S. Dhesi, R. Fan, P. Bencok, and P. Steadman, Appl. Phys. Lett. 105, 032407 (2014).

23. Z. Tian, C. Zhu, Y. Liu, J. Shi, Z. Ouyang, Z. Xia, G. Du, and S. Yuan, J. Appl. Phys. 115, 083902 (2014).

24. L. H. Bennett, R. D. McMichael, L. I. Swartzendruber, S. Hua, D. S. Lashmore, A. J. Shapiro, V. S. Gornakov, L. M. Dedukh, and V. I. Nikitenko, Appl. Phys. Lett. 66, 888 (1995).

25. M. A. Lebyodkin, N. P. Kobelev; Y. Bougherira, D. Entemeyer, C. Fressengeas, V. S. Gomakov, T.A. Lobedkina, and I. V. Shashkov, Acta Mater. 60, 3729 (2012).

26. L. V. Shashkov, M.A. Lebyodkin, and T.A. Lebedkina, Acta Mater. 60, 6842 (2012).

27. M. A. Lebyodkin, I. V. Shashkov, T. A. Lebedkina, and V. S. Gornakov, Phys. Rev. E 95, 032910 (2017).

28. M. A. Lebyodkin, T. A. Lebedkina, L.V. Shashkov, and V. S. Gornakov, Appl. Phys. Lett. 111, 032407 (2017).

29. N. N. Hsu and F. R. Breckenridge, Mater. Eval. 39, 60 (1981).

30. H. Lamb, Proc. R. Soc. London, Ser.A 93, 114 (1917).

31. I. A. Viktorov, Rayleigh and Lamb Waves: Physical Theory and Applications (Plenum, New York, 1967).

32. S. V. Kuenetsov, Acoust. Phys. 60, 95 (2014).

33. J. Slack, J. Appl. Phys. 31, 1571 (1960). 